This is an electronic reprint of the original article. This reprint may differ from the original in pagination and typographic detail.

Author(s): Puurtinen, Mikael; Elo, Merja; Jalasvuori, Matti; Kahilainen, Aapo; Ketola, Tarmo; Kotiaho, Janne Sakari; Mönkkönen, Mikko; Pentikäinen, Olli

Title: $\quad$ Temperature-dependent mutational robustness can explain faster molecular evolution at warm temperatures, affecting speciation rate and global patterns of species diversity

Year: $\quad 2016$

Version:

Please cite the original version:

Puurtinen, M., Elo, M., Jalasvuori, M., Kahilainen, A., Ketola, T., Kotiaho, J. S., Mönkkönen, M., \& Pentikäinen, O. (2016). Temperature-dependent mutational robustness can explain faster molecular evolution at warm temperatures, affecting speciation rate and global patterns of species diversity. Ecography, 39(11), $1025-$ 1033. https://doi.org/10.1111/ecog.01948

All material supplied via JYX is protected by copyright and other intellectual property rights, and duplication or sale of all or part of any of the repository collections is not permitted, except that material may be duplicated by you for your research use or educational purposes in electronic or print form. You must obtain permission for any other use. Electronic or print copies may not be offered, whether for sale or otherwise to anyone who is not an authorised user. 


\title{
Temperature-dependent mutational robustness can explain faster molecular evolution at warm temperatures, affecting speciation rate and global patterns of species diversity
}

\author{
Mikael Puurtinen ${ }^{1,2^{*}}$, Merja Elo ${ }^{1}$, Matti Jalasvuori ${ }^{1,2}$, Aapo Kahilainen ${ }^{1,3}$, Tarmo Ketola ${ }^{1,2}$, Janne S. Kotiaho ${ }^{1,4}$, \\ Mikko Mönkkönen ${ }^{1}$, Olli T. Pentikäinen ${ }^{1,5}$ \\ Correspondence to: mikael.puurtinen@jyu.fi
}

Accepted for publication in Ecography, doi: 10.1111/ecog.01948

\begin{abstract}
Distribution of species across the Earth shows strong latitudinal and altitudinal gradients with the number of species decreasing with declining temperatures. While these patterns have been recognized for well over a century, the mechanisms generating and maintaining them have remained elusive. Here, we propose a mechanistic explanation for temperature-dependent rates of molecular evolution that can influence speciation rates and global biodiversity gradients. Our hypothesis is based on the effects of temperature and temperature-adaptation on stability of proteins and other catalytic biomolecules. First, due to the nature of physical forces between biomolecules and water, stability of biomolecules is maximal around $+20^{\circ} \mathrm{C}$ and decreases as temperature either decreases or increases. Second, organisms that have adapted to cold temperatures have evolved especially flexible (but unstable) proteins to facilitate catalytic reactions in cold, where molecular movements slow down. Both these effects should result in mutations being on average more detrimental at cold temperatures (i.e. lower mutational robustness in cold). At high temperatures, destabilizing water-biomolecule interactions, and the need to maintain structures that withstand heat denaturation, should decrease mutational robustness similarly. Decreased mutational robustness at extreme temperatures will slow down molecular evolution, as a larger fraction of new mutations will be removed by selection. Lower mutational robustness may also select for reduced mutation rates, further slowing down the rate of molecular evolution. As speciation requires the evolution of epistatic incompatibilities that prevent gene flow among incipient species, slow rate of molecular evolution at extreme temperatures will directly slow down the rate at which new species arise. The proposed mechanism can thus explain why molecular evolution is faster at warm temperatures, contributing to higher speciation rate and elevated species richness in environments characterized by stable and warm temperatures.
\end{abstract}

\footnotetext{
${ }^{1}$ Department of Biological and Environmental Science, 40014 University of Jyvaskyla, Finland.

${ }^{2}$ Centre of Excellence in Biological Interactions, 40014 University of Jyvaskyla, Finland.

${ }^{3}$ Current address: Metapopulation Research Centre, 00014 University of Helsinki, Finland

${ }^{4}$ Natural History Museum, 40014 University of Jyvaskyla, Finland.

${ }^{5}$ Nanoscience Center, 40014 University of Jyvaskyla, Finland.
} 


\section{Introduction}

The global distribution of species richness is far from random or uniform. Instead, species richness shows a number of geographical gradients across the Earth. Perhaps the most striking of these patterns is the decreasing number of species from the equator toward the poles, the latitudinal diversity gradient (LDG). LDGs have been documented for a wide variety of taxa in both aquatic and terrestrial environments (Hillebrand 2004a). Over very long time-scales, the LDG has been found to be steeper during periods characterized by colder and more seasonal climates, suggesting that climate has an important role in determining distribution of species diversity over the globe (Yasuhara et al. 2012, Mannion et al. 2014). Although a large number of hypotheses have been put forward to explain LDGs (Rohde 1992, Willig et al. 2003, Mittelbach et al. 2007), the idea that greater speed of evolution and diversification in lower (warmer) latitudes may explain current LDGs has gathered empirical support over the past few decades (Gillman and Wright 2014). This "evolutionary speed hypothesis" suggests that temperature affects the rate of speciation by influencing mutation rates and/ or average generation times (Rohde 1992, Evans and Gaston 2005, Gillman and Wright 2014).

There is ample and strong evidence that evolutionary speed, measured as rate of molecular evolution, is faster at warmer temperatures (Gillman and Wright 2014). Positive association with variables related to the functional temperature of organisms and the rate of molecular evolution has been reported across metazoa (Gillooly et al. 2005), in marine protists (Allen and Gillooly 2006), in fish (Estabrook et al. 2007, Wright et al. 2011), in amphibians (Wright et al. 2010), in aquatic turtles (Lourenco et al. 2013), in flowering plants (Davies et al. 2004), and in trees (Wright et al. 2006, Gillman et al. 2010). Associations between rates of molecular evolution and net diversification (speciation minus extinction) have been reported from birds and reptiles (Eo and DeWoody 2010, Lanfear et al. 2010) and from flowering plants (Barraclough and Savolainen 2001), but not from mammals (Goldie et al. 2011). A recent review summarizing paleoecological and phylogenetic studies concluded that "there is strong evidence that a broad range of life forms have experienced faster net rates of diversification at lower latitudes" (Gillman and Wright 2014).

In a recent study, Jetz and Fine (2012) modelled global terrestrial vertebrate richness across bioregions using environmental data from past 55 million years. For ectotherms, temperature alone explained more of the variation in current richness than any of the other variables (productivity, area, or their combination integrated over the 55 million years). For both ecto- and endotherms, the best models always included temperature. This study stresses the independent effect of temperature on terrestrial vertebrate richness, especially for ectotherms. Also studies from marine systems suggest that temperature, instead of e.g. productivity, is the most important factor explaining both current (Roy et al. 1998, Rutherford et al. 1999, Rombouts et al. 2009, Rombouts et al. 2010, Tittensor et al. 2010), but see McCallum et al. (2015)), and historical patterns of species richness (Hunt et al. 2005, Yasuhara et al. 2012). The strong and consistent 
marine LDGs are also noteworthy, as they cannot easily be explained by area, time for speciation, spatial heterogeneity, or water availability that have been proposed as mechanisms underlying terrestrial LDGs (Hillebrand 2004a, Hillebrand 2004b).

However, the mechanisms by which temperature accelerates genetic evolution and diversification remain elusive (Evans and Gaston 2005). Previously, models based on the effects of body size and temperature on metabolic rate have been proposed to explain mutation rates and global diversity patterns. The metabolic theory of ecology (MTE; Brown et al. 2004) predicts the effects of body size and temperature on metabolism through considerations of vascular distribution networks and biochemical kinetics. Allen et al. (2006) combined MTE with theory of population genetics to predict the effect of temperature on rates of genetic divergence among populations and speciation in communities by specifying population size, community size, and mutation rate as temperature-dependent parameters. The theory has been further elaborated by Stegen and co-workers (Stegen et al. 2009, Stegen et al. 2012) to incorporate the effects of temperature on ecological interactions and dynamics of resource supply. The validity of assumptions underlying MTE is nevertheless strongly contested (Price et al. 2012, Storch 2012, Glazier 2014).

A key assumption in metabolic theories for biodiversity is temperature-dependence of mutation rate, which has been suggested to be caused by elevated production of DNA-damaging oxygen radicals with higher metabolism (Martin and Palumbi 1993). However, there is no evidence for a direct link between metabolic rate and molecular evolution (Mooers and Harvey 1994, Hoffmann et al. 2004, Lanfear et al. 2007), and a direct test of the role of oxidative stress in increasing heritable mutations also failed to find support for this mechanism (Joyner-Matos et al. 2011). Furthermore, it is not clear how high somatic metabolic rate could influence germ-line mutations (Lanfear et al. 2007, Galtier et al. 2009). The production of oxygen radicals by metabolism is also expected to mostly affect mitochondrial DNA, whereas nuclear DNA should be much less affected (Martin and Palumbi 1993, Hoffmann et al. 2004). The direct effect of metabolic rate on mutations may thus be of limited importance to overall genomic mutation rates. Indeed, a recent review on molecular evolution and LDG concluded that "metabolic rate may inform little on rates of mutation or molecular evolution and we are no closer to understanding the driver of variation in the rate of molecular evolution and the formation of the latitudinal biodiversity gradient" (Dowle et al. 2013).

Mutations often occur in the process of DNA replication, and factors that increase the rate of germ line cell division, especially shorter generation times, could thus explain faster rates of molecular evolution per unit time in warmer temperatures (Rohde 1992). Shorter generation times have been associated with faster molecular evolution in birds (Mooers and Harvey 1994), mammals (Bromham et al. 1996, Welch et al. 2008) and invertebrates (Thomas et al. 2010). However, it is possible that selection against somatic mutations in long-lived organisms, and not generation time per se, is the crucial factor affecting molecular evolution (Nabholz et al. 2008, Galtier et al. 2009, Lanfear et al. 2013, Bromham et al. 2015). Although there is evidence for a statistical association between generation time and molecular evolution, data on the association between 
ambient (or physiological) temperature and generation time is scarce (McCoy and Gillooly 2008 show that higher temperatures are associated with shorter lifespans), making it difficult to evaluate the general importance of the generation time hypothesis on the association between temperature and rates of molecular evolution. On the other hand, temperature has been linked to the rate of molecular evolution even in the absence of generation time differences in e.g. hummingbirds (Bleiweiss 1998) and tropical plants (Wright et al. 2006).

There are also other factors that may affect substitution rates, but whose relationship with temperature or latitude is not well known. Such factors include the degree of sperm competition (Wong 2014), organism size (Welch et al. 2008, e.g. Bromham et al. 2015), and population size (see Dowle et al. 2013). Disentangling the effects of several, often intercorrelated, factors on molecular evolution can be done by incorporating multiple factors to the same model (see e.g. Martin and Palumbi 1993, Mooers and Harvey 1994, Welch et al. 2008, Wong 2014). It is also important to bear in mind that not all genes or mutations are expected to be equally affected by various factors. For example, sperm competition intensity is not expected to influence mitochondrial DNA evolution, since mitochondria are only inherited from females.

\section{Temperature and mutational robustness}

We suggest that the effects of temperature on mutational robustness of proteins and other catalytic biomolecules (e.g. ribozymes) may provide a mechanistic explanation for the slower rate of molecular evolution in environments where ambient temperatures are cold (high latitudes, high altitudes, deep oceans). In this paper, we focus mainly on the effects of cold temperatures on mutational robustness and molecular evolution. Our hypothesis however suggests that similar patterns should also be evident at high temperatures, and we will comment evidence for slowdown of molecular evolution at high temperatures, where appropriate.

Temperature may affect mutational robustness in two ways. First, regardless of the structural details of proteins, protein thermodynamic stability at neutral pH is maximal around room temperature (Fig. 1A). This is because the hydrophobic effect, the main force driving protein folding, is maximal at this temperature (Privalov 1990, Privalov 1997, Kumar et al. 2002). The decrease in stability at colder temperatures could be explained through formations of solvent-separated-configurations where the nonpolar protein core is hydrated by a thin layer of water (Privalov 1990, Dias 2012). A very recent study suggest that in live cells, cold-denaturation of proteins can be a serious concern at realistic physiological temperatures (Danielsson et al. 2015).

Second, and perhaps more important, proteins of organisms that have evolved to function in cold environments have evolved to be thermodynamically less stable (Fig. 1B). It is a well-established empirical fact that there is a tight association between the temperature to which an organisms is adapted to and the denaturation temperature of proteins (see e.g. Fig. 1 in Somero 1995). The lowered stability of cold-adapted 
proteins is due to structural properties like decreased core hydrophobicity, increased surface hydrophobicity, increased movement of internal groups, and reduced number of interdomain and intersubunit interactions (Feller 2003, Siddiqui and Cavicchioli 2006). The decrease in thermodynamic stability is most likely a consequence of selection for increased mechanical flexibility necessary for maintaining activity of the protein in the cold environment where molecular motions slow down (Feller and Gerday 2003, DePristo et al. 2005, Siddiqui and Cavicchioli 2006). It has even been argued that cold-adapted proteins may have reached a state that is close to the lowest possible stability, i.e. that they cannot become less stable without losing the native and active conformation (Feller and Gerday 2003). Importantly, cold adaptation does not seem to shift the temperature of maximal stability, but reduces thermodynamic stability at all temperatures (Cipolla et al. 2012) (see Fig. 1B).
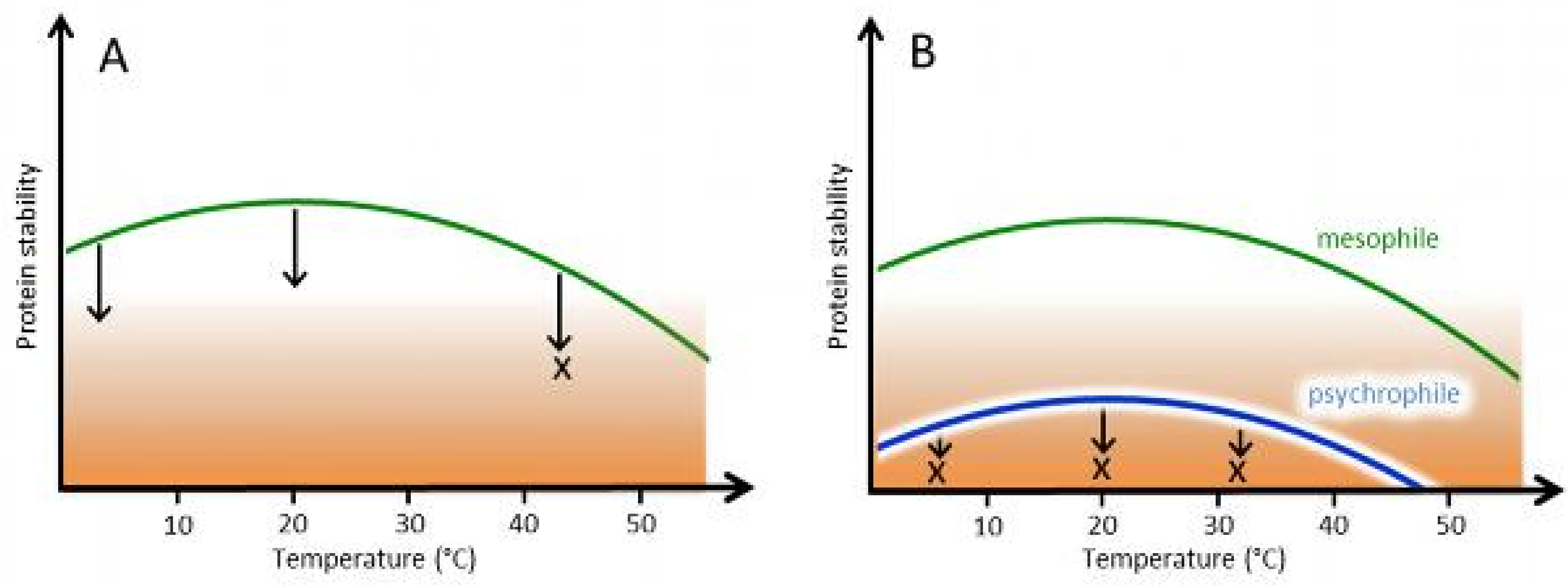

Figure 1. Stylized effects of temperature on protein stability. The intensity of the orange colour indicates the probability that a random mutation will render the protein non-functional, and the mutation will thus be removed by selection (marked with $\mathrm{x}$ ). (A) The stability maximum of most studied proteins is around $+20^{\circ} \mathrm{C}$. A destabilizing mutation may not impair protein folding and stability at $+20^{\circ} \mathrm{C}$, but at a lower (or higher) temperature the same mutation may be detrimental, and be removed by selection. A recent study (Danielsson et al. 2015) found that in live cells, protein stability decreases much more dramatically at low temperatures than depicted in this figure, suggesting that cold-denaturation is a serious concern in realistic physiological temperatures. (B) In cold-adapted organisms (psychrophiles), proteins are unstable as a consequence of selection for maintaining flexibility essential for catalysing reactions. The low stability of cold-adapted enzymes is hypothesized to result in very large proportion of mutations being harmful and being removed by selection.

Thermostability is important for molecular evolution because thermolabile proteins have lower mutational robustness. The great majority of amino-acid changing mutations are destabilizing (Tokuriki and Tawfik 2009). Mutations are likely to render a thermolabile protein non-functional because the mutated protein will not fold to its native form, or because lowered stability reduces the concentration of dissolved protein due to increased aggregation or proteolysis (Sanchez-Ruiz 2010). Misfolding proteins may even have toxic effects, 
further increasing the deleterious consequences of destabilizing mutations (Geiler-Samerotte et al. 2011). A recent computational study concluded that more thermodynamically stable proteins are more robust against random amino-acid replacements (Hormoz 2013). Thermostability has also been found to substantially increase the likelihood of proteins maintaining functionality after major restructuring (Besenmatter et al. 2007), and the likelihood of evolving new or improved functions (Bloom et al. 2006). Thermostability has further been found to decrease the harmful effect of mutagen exposure in an RNA virus (Domingo-Calap et al. 2010). At the level of amino-acid substitutions, less destabilizing substitutions are more likely to be fixed in evolving lineages (Godoy-Ruiz et al. 2004, Godoy-Ruiz et al. 2006), supporting the notion that stability effects of mutations are important in determining the rate of protein sequence evolution.

\section{Temperature effects on mutation and substitution rates}

In this section, we outline the effects of temperature on mutation and substitution rates. The logic of the argument is presented with a flow-chart in Figure 2, and in Figure 3 we give a calculated example of the relationship between temperature and key properties of mutations and substitutions, based on the formulas derived below.

In sexual organisms, mutation rate is thought to be primarily determined by the balance between reduction in the harmful effects of new mutations and the costs of more stringent proof-reading (Dawson 1998, Sniegowski et al. 2000). Dawson (1998) derived the solution for evolutionary stable mutation rate when mutations that decrease the fitness of an individual by a fraction $(1-s)$ occur at infinite number of unlinked diallelic loci, and when modifiers that decrease mutation rate directly decrease individual fitness. The solution given by Dawson (1998) for the evolutionary stable mutation rate (U*) is

$$
U^{*}=\max \left[\alpha(U)-\frac{s}{1+s} U\right],
$$

where $\alpha(\mathrm{U})$ describes the relationship between the mutation rate $\mathrm{U}$ coded by the modifier, and the direct cost (proportional reduction in fitness) of the modifier to individual fitness. A plausible explicit function for $\alpha(\mathrm{U})$ is

$$
\alpha(U)=1-e^{-U k},
$$

where $\mathrm{k}$ is a scaling factor determining the rate at which the costs of reducing the mutation rate depress individual fitness. With this function, we can write the function for $\mathrm{U}^{*}$ as

$$
U^{*}=\max \left[1-e^{-U k}-\frac{s}{1+s} U\right],
$$

where the function to be maximized is the fitness of the modifier allele, $\mathrm{w}$ : 


$$
w=1-e^{-U k}-\frac{s}{1+S} U
$$

The evolutionarily stable mutation rate in fitness-affecting loci $\mathrm{U}^{*}$ can be found by differentiating w with respect to $\mathrm{U}$

$\frac{d w}{d U}=e^{-U k} k-\frac{s}{1+s}$, and solving $\frac{d w}{d U}=0$ with respect to $\mathrm{U}$, which yields

$$
U^{*}=\frac{\ln \left(\frac{k+s k}{s}\right)}{k} .
$$

The solution (eq. 5) implies that mutation rate should evolve to lower level when new mutations are more harmful (i.e., when s increases). Our hypothesis suggests that mutations are more harmful in cold environments and in organisms with low functional temperatures, due to both the direct effect of temperature to molecule stability and the evolved lower thermostability of cold-adapted proteins, and this will result in reduced mutation rate in cold temperatures. In high temperatures, the need to maintain protein structures that are able to withstand heat denaturation should also make mutations more harmful than in benign temperatures. To illustrate the argument, let us assume that the harmfulness of mutations ( $\mathrm{s}$ ) is a function of temperature $\left(\mathrm{T}\right.$, in $\left.{ }^{\circ} \mathrm{C}\right)$, with a minimum $(\mathrm{m})$ around room temperature $\left(25^{\circ} \mathrm{C}\right.$; this value is arbitrarily chosen to yield a pattern roughly matching empirical data). Note that the relationship formalized in function 6 does not apply to any single protein, but is a heuristic relationship intended to portray the combined effects of temperature adaptation and protein-solvent interactions on mutational effects. We posit that the further temperature deviates from room temperature, the more harmful mutations become.

$$
s=\frac{(25-T)^{4}}{a}+m
$$

where a is a constant scaling factor (Fig. 3A). Substituting eq. 6 to eq. 5 and simplifying, we get the evolutionary stable mutation rate as a function of temperature (Fig. 3A):

$$
U^{*}=\frac{\ln \left\{\frac{k\left[a(1+m)+(25-T)^{4}\right]}{a m+(25-T)^{4}}\right\}}{k} .
$$

Noting that the per site mutation rate $\mu$ is proportional the genome-wide mutation rate $U$, and that the rate of neutral substitutions only depends on mutation rate (Hartl and Clark 1997), the rate of neutral substitutions (e.g. synonymous mutations that do not change the amino-acid in a protein, $\mathrm{dS}$ ) is simply proportional to the mutation rate $d S \propto \mu \propto U^{*}$ and can be modelled as

$$
d S=p U^{*}
$$


where $\mathrm{p}$ is a scaling factor to translate the genomic mutation rate to per-locus mutation rate. We can thus make the qualitative prediction that the rate of neutral substitutions will be highest at temperatures near the room temperature, and decrease as the temperature approaches $0^{\circ} \mathrm{C}$, or reaches high temperatures (Fig.3B).

The rate of substitutions with fitness effects (e.g. most non-synonymous mutations that alter the amino-acid in a protein, $\mathrm{dN}$ ) is more difficult to predict, as it depends on the distribution of mutation effects and on the effective population size (Lanfear et al. 2014). Accounting for these factors is beyond the scope of the current treatment. However, as the great majority of mutations with fitness effects are harmful (Eyre-Walker and Keightley 2007), and as the likelihood of fixation decreases rapidly as the harmfulness of mutations increases (Crow and Kimura 1970), for current purposes we can model rate substitutions at fitness-affecting loci as

$$
d N=e^{-s b} d S
$$

where $b$ is a scaling factor determining the rate of decline in fixation probability with increasing s, and thus relates to the effective population size (larger $b$ means more efficient selection, corresponding to larger effective population size) (Lanfear et al.2014). This formalization predicts that the rate of substitutions with fitness effects decreases rapidly at extreme temperatures, where mutations are more harmful (Fig. 3B).

The idea that the harmfulness of mutations depends on temperature, and is reflected in mutation and substitution rates, has been put forward before in the context of slow molecular evolution in birds and thermophiles. Prager et al. (1974) noted that birds seem to have a slower rate of molecular evolution than other vertebrates, and suggested that this might be explained by the high body temperature of birds. Since then, several studies have confirmed that molecular evolution is slower in birds than mammals, but faster than in ectothermic vertebrates. Stanley and Harrison (1999), studying cytochrome b evolution, showed that selection against nonsynonymous mutations is stronger in birds than in mammals. These findings give strong support for the hypothesis that high body temperatures in birds impose strict requirements for proteins and lead to reduced tolerance for amino acid replacements.

In thermophiles, anecdotal evidence that many missense mutations (mutations that change the amino-acid in a protein) are well tolerated in standard growth temperatures but become lethal at elevated temperatures lead Drake (2009) to formulate the "hypothesis of dangerous missense": the average missense mutation harms thermophiles more than mesophiles. In accordance with this hypothesis, Friedman et al. (2004) found that the ratio of non-synonymous to synonymous mutations ( $\mathrm{dN} / \mathrm{dS}$ ) was lower in thermophilic than in mesophilic microbes, and attributed this finding to stronger purifying selection against amino-acid changing mutations at high temperatures. Drake (2009) found that the rate of spontaneous mutation is lower in thermophiles than in mesophiles, and suggested that the lower mutation rate is an adaptation to the more harmful effects of mutations in thermophiles. However, as many studies do not include thermophiles to the data (e.g. Gillooly et al. 2005, Gillooly et al. 2007), and do not consider models where increasing temperature 
could slow molecular evolution down at high (above $40^{\circ} \mathrm{C}$ ) temperatures, it is not surprising that the phenomenon is not widely acknowledged.

Our hypothesis suggests that like high temperatures, also low temperatures lead to reduced mutational robustness (Fig. 3C), potentially explaining why rates of molecular evolution and speciation are slower in in cold environments (e.g. at high latitudes and altitudes. We are aware of two datasets that can inform about the effect of colder temperatures on strength of selection against non-synonymous mutations. Gillooly et al. (2007) showed that temperature speeds up both synonymous and non-synonymous evolution over a diverse set of animal taxa (ranging from psychrophiles to mesophiles). Although not reported in their original manuscript, the relationship between temperature and $\mathrm{dN} / \mathrm{dS}$ can be easily carried out from data published with the manuscript. Intriguingly, data suggests that selection against amino-acid changing mutations in cytochrome $b$ is indeed stronger in colder temperatures (Pearson correlation between temperature and $d N / d S ; r=0.426, n=48, P=0.003$ ). For the other studied gene (nicotinamide adenine dinucleotide), there was a non-significant relationship to the opposite direction $(r=0.311, n=36, P=0.065)$. Wright et al. (2011) studied cytochrome $b$ evolution in marine fishes, and did not find a significant association between temperature and dN/ dS. More empirical research is clearly needed to assess the effect of cold temperatures on selection against non-synonymous mutations, and consequent selection for reduced mutation rates.

However, it must be noted that synonymous changes may not all be neutral (Shabalina et al. 2013) as certain codons can serve multiple functions by, for example, coding for an amino acid while simultaneously acting as a binding site for transcriptional regulators (Stergachis et al. 2013). Furthermore, organisms can prefer some codons over others. The usage of synonymous codons appears to follow the proportions of available transfer RNA molecules in the cell, and suboptimal codon usage is likely to select for mutations that re-establish the codon-tRNA balance (Qian et al. 2012). If the concentrations of tRNAs are known, the mutation rate towards balance could be measured by comparing different types of recently acquired genes. For some genes, this rate may be necessary to be taken into account when $\mathrm{dN} / \mathrm{dS}$ is determined. 


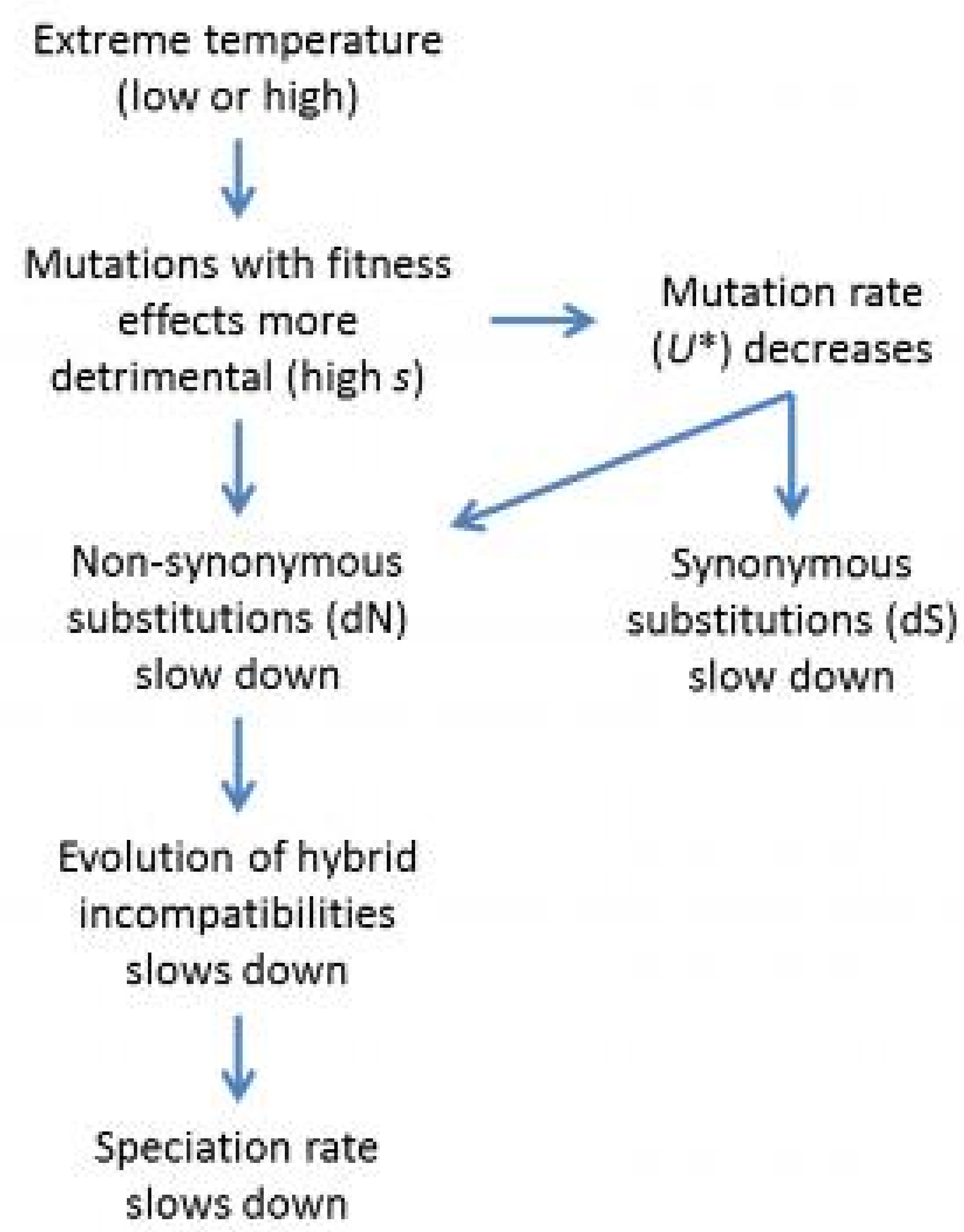

Figure 2. The effects of temperature on mutation and speciation rates. At extreme temperatures (low or high), mutations with fitness effects are more detrimental, which directly slows down evolution at sites that affect fitness (e.g. non-synonymous substitutions, $\mathrm{dN}$ ). Because mutations are on average more detrimental at extreme temperatures, selection favours reduced mutation rate, and slows down evolution at sites without fitness affects as well (e.g. synonymous substitutions, dS). Slower mutation rate further slows down evolution at sites that affect fitness. Slow evolution of protein-coding sequences means that genetic incompatibilities between isolated populations evolve at a slower rate, reducing the rate at which new species form. 

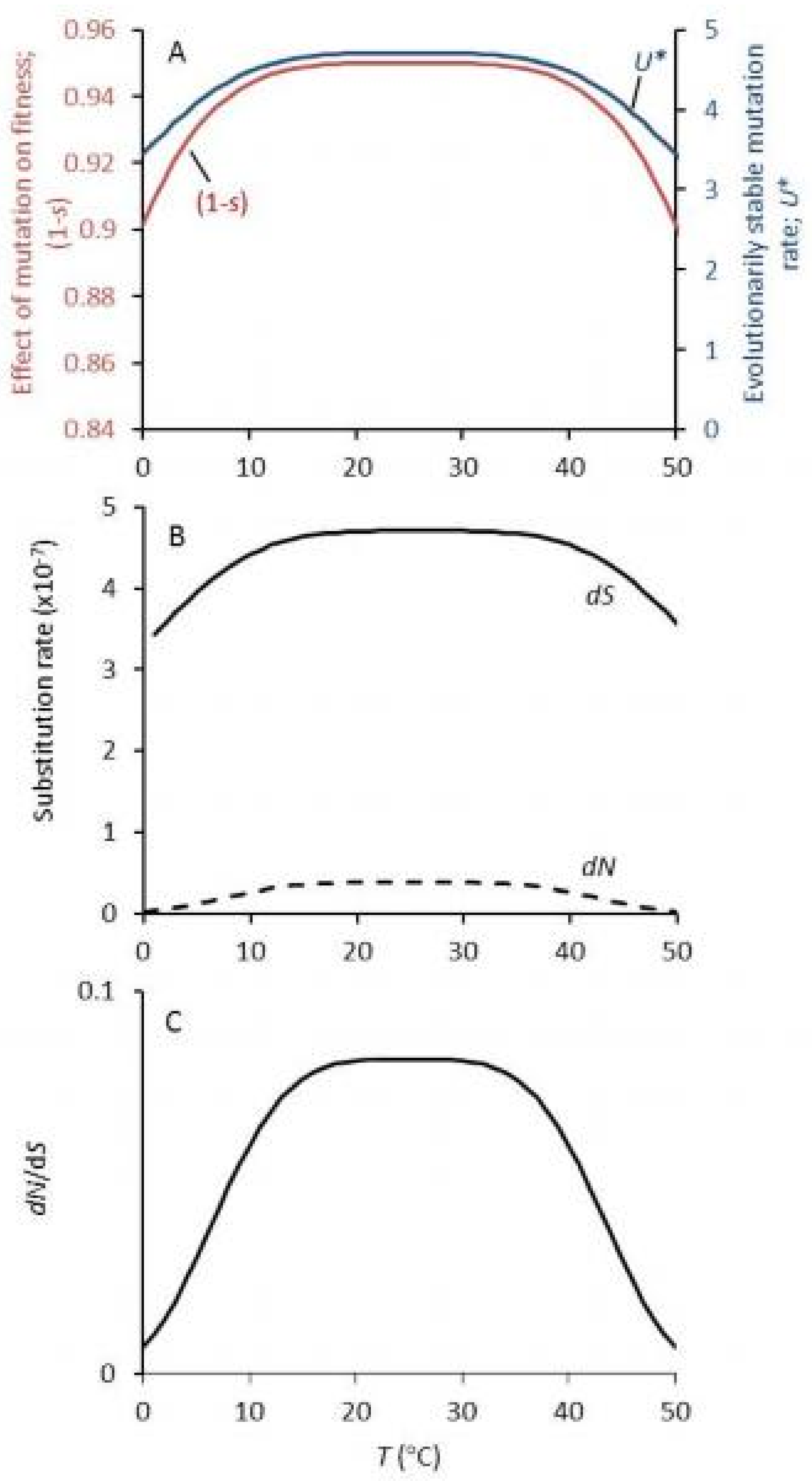

Figure 3. Effects of temperature on mutation and substitution rates. A. We hypothesize that mutations will be more detrimental in both low and high temperatures (red line, left axis), and this will select for reduced mutation rates at extreme temperatures (blue line, right axis; eq. 7). B. The synonymous substitution rate (dS; continuous line) mirrors the mutation rate (eq. 8), but the non-synonymous substitution rate (dN; dashed line) is depressed at extreme temperatures, due to the more intense purifying selection (eq. 9). C. The ratio of substitution rates at coding and non-coding loci shows strong effects of temperature, being positive at the cold temperatures and negative at high temperatures. Values used in the calculations: $k=0.5 ; a=8 \times 10^{6} ; m=0.05$; $p=10^{-7} ; b=50$. 


\section{Operative temperatures in ecto- and endotherms}

The operative temperatures of organisms are of central importance to our hypothesis. For terrestrial ectotherms that can seek shade to avoid direct sunlight, maximal operative temperatures show little latitudinal gradient from the equator to latitudes up to 50 degrees, being around $25^{\circ} \mathrm{C}$ to $30^{\circ} \mathrm{C}$. However, minimal operative temperatures decline from around $22^{\circ} \mathrm{C}$ near the equator to $0^{\circ} \mathrm{C}$ or below at higher latitudes (see Figure 4 in Sunday et al. 2014). At higher latitudes, proteins therefore need to operate and be stable in both cold and warm environments. We suggest that within the range of operational temperatures encountered by organisms in current global climate (excluding exposed or day-active organisms in hot deserts), minimal operational temperatures exert the strongest selection against non-synonymous mutations and explain the slower rate of molecular evolution in ectotherms at high altitudes and latitudes

A number of studies have found slower rates of molecular evolution at cold ambient temperatures also in endotherms. Bleiweiss (1998) showed that the rate of DNA evolution in hummingbirds decreases with altitude. More recent studies have reported slower cytochrome b evolution at higher latitudes and altitudes in mammals (Gillman et al. 2009) and in birds (Gillman et al. 2012). At first sight, these findings would seem to suggest that some other factor than temperature must drive the rate of molecular evolution, since endotherms can control their body temperature. However, many birds and mammals reduce their body temperature close to ambient temperatures to conserve energy during adverse (cold) conditions by going into torpor. Low torpor body temperatures were speculated as one possible cause for the observed slowdown of molecular evolution by Bleiweiss (1998) and Gillman et al. (2009). We suggest that cold torpor selects for cold-active proteins that facilitate heating up the body from torpor to active temperature; see e.g. Williams et al. (2011) for rapid changes in the core body temperature of a hibernating ground squirrel. Hence, like psychrophiles, endotherms that regularly go into cold torpor may have evolved thermolabile proteins to enhance catalytic activity in cold, and this has coincidentally increased mutational sensitivity. In mammals, temporal variation in body temperatures increases with increasing latitude and decreasing average temperature (Boyles et al. 2013), supporting the suggestion that endotherm functional temperatures can follow environmental temperature clines.

Although we suggest that minimal rather than maximal operational temperatures are more important in limiting rates of molecular evolution within the conditions of current climate (excluding hot deserts), the situation may be very different if global climate was much warmer. Elevated temperatures exert stronger boundaries to organismal survival than low temperatures, possibly because of the destabilizing effects of heat on proteins (Araujo et al. 2013). If global temperatures were to increase significantly, we could thus expect diversity to decline in warmest regions, both via extinction and possibly also via slowdown in molecular evolution. Indeed, during the extreme greenhouse conditions ca. $250 \mathrm{Mya}$, equatorial regions were devoid of most terrestrial and marine taxa present in other parts of the world at that time (Sun et al. 2012) 


\section{Genetics of speciation}

Completion of speciation requires the evolution of epistatic genetic incompatibilities that prevent gene flow between incipient species by causing hybrid inviability or infertility. Such incompatibilities arise most easily in populations that are reproductively isolated. As originally explained by Bateson, Dobzhansky, and Muller, mutations that are adaptive or neutral in the population they originated in can be functionally incompatible when brought together in cross-population hybrids (Presgraves 2010a). Recently, molecular studies have begun to reveal the exact identities and functions of some such hybrid incompatibility genes. The great majority of the identified loci are protein-coding genes (Presgraves 2010b, Presgraves 2010a). If the rate of amino-acid substitutions is lower in cold temperatures, as we hypothesize, cold temperatures will slow down the speciation rate. Speciation rate will slow down irrespective whether the loci underlying incompatibility evolve neutrally, or whether they are under positive selection, e.g. due to intragenomic or intersexual conflicts (Presgraves 2010a, Crespi and Nosil 2013), or due to environmental selective pressures. Slower mutation rate will also translate to lower standing epistatic genetic variation, meaning that there is less variation that can potentially evolve to hybrid incompatibilities between isolated populations (Cutter 2012, Corbett-Detig et al. 2013).

\section{Assessing the validity of different hypotheses}

We have proposed a mechanism that can explain the strong and consistent associations between temperature, molecular evolution, diversification, and global patterns of species diversity. Our suggested mechanism is based on general biophysical properties of proteins and other biomolecules (like ribozymes), and hence applies to all organisms regardless of the type of environment or trophic position. Currently the most popular hypothesis for explaining the association between temperature and molecular evolution is the Metabolic Theory of Ecology (MTE; Brown et al. 2004, Gillooly et al. 2005, Allen and Gillooly 2006, Gillooly et al. 2007), which proposes that mass-specific metabolic rates are linearly and positively associated with rates of molecular evolution. Table 1 lists a number of predictions that differ between MTE and our temperaturedependent mutational robustness hypothesis, allowing for comparison of the two hypotheses. Our hypothesis is based on the unique assumption that homologous proteins from organisms from cold (or hot) temperatures will be more sensitive to random amino-acid substitutions at operative temperatures than proteins from mesophilic organisms. Testing this assumption will be crucial for assessing the validity of the mechanism proposed in this article. 
Table 1. Differing predictions for patterns between temperature and molecular evolution between the metabolic theory of ecology and the temperature-dependent mutational robustness hypothesis presented in this article. See main text for data bearing on these predictions and further discussion.

\begin{tabular}{|c|c|c|}
\hline & $\begin{array}{l}\text { Metabolic theory of ecology } \\
\text { (MTE) }\end{array}$ & $\begin{array}{l}\text { Temperature-dependent } \\
\text { mutational robustness } \\
\text { hypothesis }\end{array}$ \\
\hline $\begin{array}{l}\text { Relationship between } \\
\text { temperature and per } \\
\text { generation mutation rate in } \\
\text { ectotherms }\end{array}$ & $\begin{array}{l}\text { No relationship (Gillooly et al. } \\
2005 \text {, Gillooly et al. 2007) }\end{array}$ & $\begin{array}{l}\text { Hump-shaped (increasing from } \\
\text { psycrophiles to mesophiles; } \\
\text { decreasing from mesophiles to } \\
\text { thermophiles) }\end{array}$ \\
\hline $\begin{array}{l}\text { Relationship between } \\
\text { operative temperature and } \\
\text { dN/ dS (lower dN/ dS indicates } \\
\text { stronger purifying selection) }\end{array}$ & $\begin{array}{l}\text { No relationship (Gillooly et al. } \\
2007 \text { ) }\end{array}$ & $\begin{array}{l}\text { Hump-shaped from } \\
\text { psycrophiles to thermophiles. }\end{array}$ \\
\hline $\begin{array}{l}\text { Relationship between } \\
\text { operative temperature and rate } \\
\text { of synonymous mutations (per } \\
\text { time unit) }\end{array}$ & $\begin{array}{l}\text { Increasing, practically linear } \\
\text { (after controlling for body } \\
\text { mass }^{-0.25} \text { ). }\end{array}$ & $\begin{array}{l}\text { Hump-shaped from } \\
\text { psycrophiles to thermophiles } \\
\text { (after controlling for possible } \\
\text { effect of generation time). }\end{array}$ \\
\hline $\begin{array}{l}\text { Relationship between } \\
\text { environmental temperature } \\
\text { and rate of molecular evolution } \\
\text { in endotherms }\end{array}$ & $\begin{array}{l}\text { For organism of equal size, } \\
\text { molecular evolution should be } \\
\text { faster in cold environments } \\
\text { since active metabolic rate } \\
\text { increases with decreasing } \\
\text { temperature (Anderson and } \\
\text { Jetz 2005, Clarke et al. 2010). }\end{array}$ & $\begin{array}{l}\text { Slower in cold environments } \\
\text { (for species with cold torpor), } \\
\text { since cold-active enzymes are } \\
\text { less mutationally robust and } \\
\text { thus evolve more slowly. }\end{array}$ \\
\hline
\end{tabular}

\section{References}

Allen, A. P. and Gillooly, J. F. 2006. Assessing latitudinal gradients in speciation rates and biodiversity at the global scale. - Ecol. Lett. 9: 947-954.

Allen, A. P. et al. 2006. Kinetic effects of temperature on rates of genetic divergence and speciation. - P. Natl. Acad. Sci. USA 103: 9130-9135.

Anderson, K.J. and Jetz, W. 2005. The broad-scale ecology of energy expenditure of endotherms. - Ecol. Lett. 8: 310-318.

Araujo, M. B. et al. 2013. Heat freezes niche evolution. — Ecol Lett 16: 1206-19.

Barraclough, T. G. and Savolainen, V. 2001. Evolutionary rates and species diversity in flowering plants. Evolution 55: 677-683.

Besenmatter, W. et al. 2007. Relative tolerance of mesostable and thermostable protein homologs to extensive mutation. — Proteins Structure Function and Bioinformatics 66: 500-506.

Bleiweiss, R. 1998. Slow rate of molecular evolution in high-elevation hummingbirds. - P. Natl. Acad. Sci. USA 95: 612-616.

Bloom, J. D. et al. 2006. Protein stability promotes evolvability. — P. Natl. Acad. Sci. USA 103: 5869-5874.

Boyles, J. G. et al. 2013. A global heterothermic continuum in mammals. — Global Ecol. Biogeogr. 22: 10291039.

Bromham, L. et al. 2015. Exploring the relationships between mutation rates, life history, genome size, environment, and species richness in flowering plants. - Am. Nat. 185: 507-524.

Bromham, L. et al. 1996. Determinants of rate variation in mammalian DNA sequence evolution. - Journal of Molecular Evolution 43: 610-621.

Brown, J. H. et al. 2004. Toward a metabolic theory of ecology. — Ecology 85: 1771-1789. 
Cipolla, A. et al. 2012. Temperature adaptations in psychrophilic, mesophilic and thermophilic chloridedependent alpha-amylases. — Biochimie 94: 1943-1950.

Clarke, A. et al. 2010. Scaling of basal metabolic rate with body mass and temperature in mammals. - Journal of Animal Ecology 79: 610-619.

Corbett-Detig, R. B. et al. 2013. Genetic incompatibilities are widespread within species. — Nature 504: 135137.

Crespi, B. and Nosil, P. 2013. Conflictual speciation: Species formation via genomic conflict. — Trends Ecol. Evol. 28: 48-57.

Crow, J. F. and Kimura, M. 1970. An introduction to population genetics theory. - Harper \& Row.

Cutter, A. D. 2012. The polymorphic prelude to bateson-dobzhansky-muller incompatibilities. - Trends Ecol. Evol. 27: 209-218.

Danielsson, J. et al. 2015. Thermodynamics of protein destabilization in live cells. - P. Natl. Acad. Sci. USA

Davies, T. J. et al. 2004. Environmental energy and evolutionary rates in flowering plants. - Proceedings of the Royal Society of London B Biological Sciences 271:2195-2200.

Dawson, K. J. 1998. Evolutionarily stable mutation rates. - J. Theor. Biol. 194: 143-157.

DePristo, M. A. et al. 2005. Missense meanderings in sequence space: A biophysical view of protein evolution. - Nat. Rev. Genet. 6: 678-87.

Dias, C. L. 2012. Unifying microscopic mechanism for pressure and cold denaturations of proteins. - Phys. Rev. Lett. 109:

Domingo-Calap, P. et al. 2010. Selection for thermostability can lead to the emergence of mutational robustness in an rna virus. - J. Evol. Biol. 23: 2453-2460.

Dowle, E. J. et al. 2013. Molecular evolution and the latitudinal biodiversity gradient. - Heredity 110: 501-10.

Drake, J. W. 2009. Avoiding dangerous missense: Thermophiles display especially low mutation rates. — PLoS Genet. 5: e1000520.

Eo, S. H. and DeWoody, J. A. 2010. Evolutionary rates of mitochondrial genomes correspond to diversification rates and to contemporary species richness in birds and reptiles. - Proceedings of the Royal Society Biological Sciences Series B 277: 3587-3592.

Estabrook, G. F. et al. 2007. Body mass and temperature influence rates of mitochondrial DNA evolution in north american cyprinid fish. - Evolution 61: 1176-1187.

Evans, K. L. and Gaston, K. J. 2005. Can the evolutionary-rates hypothesis explain species-energy relationships? - Funct. Ecol. 19: 899-915.

Eyre-Walker, A. and Keightley, P. D. 2007. The distribution of fitness effects of new mutations. - Nat. Rev. Genet. 8: 610-618.

Feller, G. 2003. Molecular adaptations to cold in psychrophilic enzymes. — CMLS Cellular and Molecular Life Sciences 60: 648-662.

Feller, G. and Gerday, C. 2003. Psychrophilic enzymes: Hot topics in cold adaptation. - Nat. Rev. Microbiol. 1: 200-208.

Friedman, R. et al. 2004. Genome-wide patterns of nucleotide substitution reveal stringent functional constraints on the protein sequences of thermophiles. - Genetics 167: 1507-1512.

Galtier, N. et al. 2009. Mitochondrial whims: Metabolic rate, longevity and the rate of molecular evolution. Biol. Lett. 5: 413-416.

Geiler-Samerotte, K. A. et al. 2011. Misfolded proteins impose a dosage-dependent fitness cost and trigger a cytosolic unfolded protein response in yeast. - P. Natl. Acad. Sci. USA 108: 680-685.

Gillman, L. N. et al. 2010. Faster evolution of highly conserved DNA in tropical plants. - J. Evol. Biol. 23: 13271330.

Gillman, L. N. et al. 2009. Latitude, elevation and the tempo of molecular evolution in mammals. - P. R. Soc. B. 276: 3353-3359.

Gillman, L. N. et al. 2012. The tempo of genetic evolution in birds: Body mass and climate effects. - J. Biogeogr. 39: 1567-1572.

Gillman, L. N. and Wright, S. D. 2014. Species richness and evolutionary speed: The influence of temperature, water and area. - J. Biogeogr. 41: 39-51.

Gillooly, J. F. et al. 2005. The rate of DNA evolution: Effects of body size and temperature on the molecular clock. - P. Natl. Acad. Sci. USA 102: 140-145.

Gillooly, J. F. et al. 2007. Effects of metabolic rate on protein evolution. — Biol. Lett. 3: 655-659. 
Glazier, D. S. 2014. Is metabolic rate a universal 'pacemaker' for biological processes? - Biol. Rev. Camb. Philos. Soc. doi: 10.1111/ brv.12115:

Godoy-Ruiz, R. et al. 2006. Natural selection for kinetic stability is a likely origin of correlations between mutational effects on protein energetics and frequencies of amino acid occurrences in sequence alignments. - J. Mol. Biol. 362: 966-978.

Godoy-Ruiz, R. et al. 2004. Relation between protein stability, evolution and structure, as probed by carboxylic acid mutations. - J. Mol. Biol. 336: 313-318.

Goldie, X. et al. 2011. Diversification and the rate of molecular evolution: No evidence of a link in mammals. BMC Evol. Biol. 11: 286.

Hartl, D. L. and Clark, A. G. 1997. Principles of population genetics. - Sinauer Associates, Inc.

Hillebrand, H. 2004a. On the generality of the latitudinal diversity gradient. - Am. Nat. 163: 192-211.

Hillebrand, H. 2004b. Strength, slope and variability of marine latitudinal gradients. - Mar. Ecol. Prog. Ser. 273: 251-267.

Hoffmann, S. et al. 2004. Reactive oxygen species derived from the mitochondrial respiratory chain are not responsible for the basal levels of oxidative base modifications observed in nuclear DNA of mammalian cells. — Free Radical Biology and Medicine 36: 765-773.

Hormoz, S. 2013. Amino acid composition of proteins reduces deleterious impact of mutations. - Sci. Rep. 3: 2919.

Hunt, G. et al. 2005. Species-energy relationship in the deep sea: A test using the quaternary fossil record. Ecol. Lett. 8: 739-747.

Jetz, W. and Fine, P. V. A. 2012. Global gradients in vertebrate diversity predicted by historical areaproductivity dynamics and contemporary environment. — PLoS Biol 10: e1001292.

Joyner-Matos, J. et al. 2011. No evidence of elevated germline mutation accumulation under oxidative stress in caenorhabditis elegans. - Genetics 189: 1439-1447.

Kumar, S. et al. 2002. Maximal stabilities of reversible two-state proteins. — Biochemistry 41: 5359-5374.

Lanfear, R. et al. 2013. Taller plants have lower rates of molecular evolution. - Nat. Commun. 4: 1879.

Lanfear, R. et al. 2010. Mutation rate is linked to diversification in birds. - P. Natl. Acad. Sci. USA 107: 2042320428.

Lanfear, R. et al. 2014. Population size and the rate of evolution. - Trends Ecol. Evol. 29: 33-41.

Lanfear, R. et al. 2007. Metabolic rate does not calibrate the molecular clock. - P. Natl. Acad. Sci. USA 104: 15388-15393.

Lourenco, J. M. et al. 2013. The determinants of the molecular substitution process in turtles. - J. Evol. Biol. 26: $38-50$.

Mannion, P. D. et al. 2014. The latitudinal biodiversity gradient through deep time. - Trends Ecol. Evol. 29: 42-50.

Martin, A. P. and Palumbi, S. R. 1993. Body size, metabolic-rate, generation time, and the molecular clock. — P. Natl. Acad. Sci. USA 90: 4087-4091.

McCallum, A. W. et al. 2015. Productivity enhances benthic species richness along an oligotrophic indian ocean continental margin. - Global Ecol. Biogeogr. 24: 462-471.

McCoy, M. W. and Gillooly, J. F. 2008. Predicting natural mortality rates of plants and animals. - Ecol. Lett. 11: 710-716.

Mittelbach, G. G. et al. 2007. Evolution and the latitudinal diversity gradient: Speciation, extinction and biogeography. - Ecol. Lett. 10: 315-331.

Mooers, A. Ø. and Harvey, P. H. 1994. Metabolic rate, generation time, and the rate of molecular evolution in birds. - Molecular phylogenetics and evolution 3: 344-350.

Nabholz, B. et al. 2008. Strong variations of mitochondrial mutation rate across mammals-the longevity hypothesis. - Mol. Biol. Evol. 25: 120-130.

Prager, E. M. et al. 1974. Slow evolution of transferrin and albumin in birds according to micro-complement fixation analysis. - Journal of molecular evolution 3: 243-262.

Presgraves, D. C. 2010a. Darwin and the origin of interspecific genetic incompatibilities. - Am. Nat. 176: S45S60.

Presgraves, D. C. 2010b. The molecular evolutionary basis of species formation. - Nat. Rev. Genet. 11: 175180.

Price, C. A. et al. 2012. Testing the metabolic theory of ecology. - Ecol. Lett. 15: 1465-74.

Privalov, P. L. 1990. Cold denaturation of proteins. — Crit. Rev. Biochem. Mol. 25: 281-305. 
Privalov, P. L. 1997. Thermodynamics of protein folding. - J. Chem. Thermodyn. 29: 447-474.

Qian, W. et al. 2012. Balanced codon usage optimizes eukaryotic translational efficiency. - PLoS Genet. 8: e1002603.

Rohde, K. 1992. Latitudinal gradients in species diversity: The search for the primary cause. - Oikos 514-527.

Rombouts, I. et al. 2009. Global latitudinal variations in marine copepod diversity and environmental factors. - P. R. Soc. B. 276: 3053-3062.

Rombouts, I. et al. 2010. A multivariate approach to large-scale variation in marine planktonic copepod diversity and its environmental correlates. - Limnol. Oceanogr. 55: 2219-2229.

Roy, K. et al. 1998. Marine latitudinal diversity gradients: Tests of causal hypotheses. - P. Natl. Acad. Sci. USA 95: 3699-3702.

Rutherford, S. et al. 1999. Environmental controls on the geographic distribution of zooplankton diversity. Nature 400: 749-753.

Sanchez-Ruiz, J. M. 2010. Protein kinetic stability. — Biophys. Chem. 148: 1-15.

Shabalina, S. A. et al. 2013. Sounds of silence: Synonymous nucleotides as a key to biological regulation and complexity. - Nucleic Acids Res. 41: 2073-2094.

Siddiqui, K. S. and Cavicchioli, R. 2006. Cold-adapted enzymes. — Annu. Rev. Biochem. 75: 403-433.

Sniegowski, P. D. et al. 2000. The evolution of mutation rates: Separating causes from consequences. Bioessays 22: 1057-1066.

Somero, G. N. 1995. Proteins and temperature. - Annu. Rev. Physiol. 57: 43-68.

Stanley, S. E. and Harrison, R. G. 1999. Cytochrome b evolution in birds and mammals: An evaluation of the avian constraint hypothesis. - Mol. Biol. Evol. 16: 1575-1585.

Stegen, J. C. et al. 2009. Advancing the metabolic theory of biodiversity. — Ecol. Lett. 12: 1001-15.

Stegen, J. C. et al. 2012. Evolving ecological networks and the emergence of biodiversity patterns across temperature gradients. — Proceedings of the Royal Society Biological Sciences Series B 279: 10511060 .

Stergachis, A. B. et al. 2013. Exonic transcription factor binding directs codon choice and affects protein evolution. - Science 342: 1367-1372.

Storch, D. 2012. Biodiversity and its energetic and thermal controls. — In: Sibly, R. M. et al. (eds), Metabolic ecology: A scaling approach. John Wiley \& Sons, Ltd.

Sun, Y. D. et al. 2012. Lethally hot temperatures during the early triassic greenhouse. — Science 338: 366-370.

Sunday, J. M. et al. 2014. Thermal-safety margins and the necessity of thermoregulatory behavior across latitude and elevation. - P. Natl. Acad. Sci. USA 111: 5610-5615.

Thomas, J. A. et al. 2010. A generation time effect on the rate of molecular evolution in invertebrates. - Mol. Biol. Evol. 27: 1173-1180.

Tittensor, D. P. et al. 2010. Global patterns and predictors of marine biodiversity across taxa. — Nature 466: 1098-U107.

Tokuriki, N. and Tawfik, D. S. 2009. Stability effects of mutations and protein evolvability. - Curr. Opin. Struc. Biol. 19: 596-604.

Welch, J.J. et al. 2008. Correlates of substitution rate variation in mammalian protein-coding sequences. BMC Evol. Biol. 8: 53.

Williams, C. T. et al. 2011. Data logging of body temperatures provides precise information on phenology of reproductive events in a free-living arctic hibernator. - Journal of Comparative Physiology B Biochemical Systemic and Environmental Physiology 181:1101-9.

Willig, M. R. et al. 2003. Latitudinal gradients of biodiversity: Pattern, process, scale, and synthesis. — Annu. Rev. Ecol. Evol. S. 34: 273-309.

Wong, A. 2014. Covariance between testes size and substitution rates in primates. - Mol. Biol. Evol. 31: 14321436.

Wright, S. et al. 2006. The road from santa rosalia: A faster tempo of evolution in tropical climates. - P. Natl. Acad. Sci. USA 103: 7718-7722.

Wright, S. et al. 2011. Thermal energy and the rate of genetic evolution in marine fishes. - Evol. Ecol. 25: 525530.

Wright, S. D. et al. 2010. Energy and the tempo of evolution in amphibians. — Global Ecol. Biogeogr. 19: 733740.

Yasuhara, M. et al. 2012. Latitudinal species diversity gradient of marine zooplankton for the last three million years. - Ecol. Lett. 15: 1174-1179. 
Acknowledgements - We thank Adam Algar, Folmer Bokma, Richard Field, and Jean-Michel Roberge for contributing to the workshop on energy and biodiversity in Kotiaho mansion in 2011. Funding has been provided by Academy of Finland (for MP and ME) and by Kone Foundation (for MP, ME and JSK). We thank the three anonymous referees for constructive comments on earlier version of the manuscript. 